\title{
IFN- $\gamma$-dependent epigenetic regulation instructs colitogenic monocyte/macrophage lineage differentiation in vivo
}

\author{
Y Nakanishi ${ }^{1,2}$, T Sato $^{1,3}, \mathrm{~K}^{\text {Takahashi }^{4}}$ and T Ohteki ${ }^{1}$
}

Colonic macrophages induce pathogenic inflammation against commensal bacteria, leading to inflammatory bowel disease (IBD). Although the ontogeny of colonic macrophages has been well studied in the past decade, how macrophages gain colitogenic properties during the development of colitis is unknown. Using a chemically induced colitis model, we showed that accumulated $\mathrm{Ly}_{6} \mathrm{C}^{+}$cells consisting of inflammatory monocytes and inflammatory macrophages strongly expressed representative colitogenic mediators such as tumor necrosis factor- $\alpha$ (TNF- $\alpha$ ) and inducible nitric oxide synthase (iNOS). The interferon- $\gamma$-signal transducer and activator of transcription 1 (IFN- $\gamma-\mathrm{Stat} 1$ ) pathway was required for generating colitogenic macrophages, given that Stat $1^{-1-}$ mice had less severe colitis and fewer colitogenic macrophages. Notably, IFN- $\gamma$ induced histone acetylation at the promoter regions of the Tnf and Nos2 loci in the monocyte and macrophage lineage, indicating that IFN- $\gamma$-dependent epigenetic regulation instructs the development of the colitogenic monocyte and macrophage lineage in vivo. Collectively, our results provide the essential mechanism by which dysregulated colitogenic monocytes/macrophages develop at the colon mucosa during inflammation, and suggest a new drug target for treating IBD.

\section{INTRODUCTION}

Macrophages, which are specialized for phagocytosis, are an essential component of the innate immune system. Recent discoveries reveal that macrophage functions are not restricted to host defense and the clearance of apoptotic cells, but also extend to tissue development, homeostasis, and repair. ${ }^{1}$ Macrophages can be generated from the yolk sac, from fetal liver, and through hematopoietic stem cell-derived hematopoiesis in the bone marrow. Most tissue macrophages are derived from embryonic precursors that are seeded in the tissues before birth and are maintained by self-renewal after birth, whereas macrophages in the heart, dermis, and gut are continuously replaced by monocyte precursors in adulthood. ${ }^{2-4}$ In addition, macrophages acquire tissue-specific characteristics through transcriptional and epigenetic regulation by environmental factors in the local tissue. ${ }^{5}$ For example, retinoic acid in the omentum, transforming growth factor- $\beta$ in the brain, and heme in the spleen induce GATA6 in peritoneal macrophages, Sall1 in microglia, and Spi-C in red-pulp macrophages, respectively. ${ }^{6-8}$

Immune responses in the colon must be tightly regulated. An invasion of pathogenic microbes into the colon induces a robust protective immune response; however, a similar response mounted against commensal bacteria if the colonic epithelial barrier breaks down can lead to inflammatory bowel disease (IBD). Unlike many tissue macrophages, colonic macrophages maintain their homeostasis from a constant supply of blood monocytes that differentiate into macrophages in parallel with their fitness to respond to commensal bacteria, ${ }^{9}$ through a process referred to as the "monocyte waterfall." macrophages and their precursor monocytes are crucial contributors to the development of the IBD pathology. A specific deletion of the interleukin-10 (IL-10) receptor on CX3CR1-positive macrophages causes spontaneous colitis. ${ }^{11}$ In

${ }^{1}$ Department of Biodefense Research, Tokyo Medical and Dental University, Tokyo, Japan. ${ }^{2}$ IBD project, Laboratory for Integrated Research Projects on Intractable Diseases, Medical Research Institute, Tokyo Medical and Dental University (TMDU), Tokyo, Japan. ${ }^{3} J a p a n$ Science and Technology Agency, Precursory Research for Embryonic Science and Technology (PRESTO), Saitama, Japan and ${ }^{4}$ College of Bioresource Sciences, Nihon University, Kanagawa, Japan. Correspondence: T Ohteki (ohteki.bre@mri.tmd.ac.jp) 
addition, we and another group reported that blockading the infiltration of inflammatory monocytes with an anti-CCR2 antibody or by depleting Gram-positive bacteria attenuates dextran sodium sulfate (DSS)-induced colitis. ${ }^{12,13}$ Asano et al. ${ }^{14}$ showed that CD169-positive macrophages in the colon are responsible for mobilizing monocytes under inflammatory conditions. These findings suggest that monocyte recruitment into the colon is a prerequisite for the development of colitis. However, little is known about the environmental factors and molecular mechanisms that promote the differentiation of inflammatory monocytes into colitogenic macrophages in vivo.

Tumor necrosis factor- $\alpha$ (TNF- $\alpha$ ) and inducible nitric oxide synthase (iNOS) are representative inflammatory mediators that contribute to the development of colitis, suggesting that a blockade of these mediators may be therapeutic. In fact, the apoptosis of intestinal epithelial cells was reduced in IL-10 ${ }^{-1-}$ colitic mice treated with iNOS inhibitors and a TNFneutralizing monoclonal antibody. ${ }^{15}$ We previously reported that accumulations of $\mathrm{CD}_{11 \mathrm{~b}}{ }^{+} \mathrm{CD} 11 \mathrm{c}^{\mathrm{int} /-}$ cells in the inflamed colon contain macrophages, monocytes, eosinophils, and neutrophils at the peak of inflammation; of these, only the monocytes and macrophages produce the colitogenic mediators TNF and iNOS. ${ }^{13}$ Inflammation switches monocytes from differentiating into anti-inflammatory macrophages to inflammatory macrophages and dendritic cells. ${ }^{12,16-18}$ However, the identity and detailed differentiation mechanisms of colitogenic as well as anti-inflammatory macrophages in vivo are poorly understood. Here, using a DSS-induced colitis model, we found that the production of colitogenic mediators was confined to inflammatory monocyte and inflammatory macrophage subpopulations among $\mathrm{CD} 64^{+}$cells, suggesting that these cells are colitogenic monocyte/macrophages. In this context, interferon$\gamma$ (IFN- $\gamma$ )-dependent signal transducer and activator of transcription 1 (Stat1) activation was required for the development of colitogenic macrophages from monocytes. Importantly, the IFN- $\gamma$-dependent Stat1 activation enhanced the acetylation of histone at the promoter regions of colitogenic mediators in the colitogenic monocytes/macrophages.

\section{RESULTS}

\section{Identification of colitogenic monocyte/macrophage subpopulations}

The colon lamina propria contains a variety of immune cells, including monocytes, macrophages, and dendritic cells. The "monocyte waterfall," a recently proposed model for intestinal macrophage differentiation, discriminates between resident macrophages, monocyte-derived macrophages, and dendritic cells. ${ }^{10,12,17,18}$ According to this model, after gating on live $\mathrm{CD}^{+} 5^{+}$SSC $^{\text {low }} \mathrm{CD} 3 \varepsilon^{-} \mathrm{CD}_{19}{ }^{-} \mathrm{NK}_{1.1^{-}}$Ter119 $^{-}$Ly6G $^{-}$ $\mathrm{CD}_{11 \mathrm{~b}^{+}}$cells in the inflamed colon (Supplementary Figure S1A online), we divided the $\mathrm{CD} 64^{\text {low/ }}+$ cells into four subpopulations based on the expression of Ly6C, major histocompatibility complex class-II (MHC-II), and CD206 (macrophage mannose receptor (MMR)), a representative marker for resident macrophages. ${ }^{12}$ Ly6C ${ }^{+}$cells contained MHC- $\mathrm{II}^{-}$inflammatory monocytes (P1) and $\mathrm{MHC}^{-\mathrm{II}^{+}}$ inflammatory macrophages (P2), whereas Ly6C ${ }^{-}$MHC- $\mathrm{II}^{+}$ cells contained $\mathrm{MMR}^{-}$migrant macrophages (P3) and $\mathrm{MMR}^{+}$resident macrophages (P4) (Figure 1a and Supplementary Figure S1B-D). Supporting these definitions, the cells in P1 were small in size and showed a typical monocyte-like morphology with a horseshoe-shaped nucleus, whereas those in P4 showed a resident macrophage morphology; i.e., large with an abundant foamy cytoplasm. P2 and P3 showed intermediate morphologies between P1 and P4 (Supplementary Figure S1C). At 4 days after DSS challenge, all of the fractions expressed high levels of the chemokine receptor CCR2 (Supplementary Figure S1C), indicating that they were of the monocyte/macrophage lineage. Consistent with these observations, the expression level of CX3CR1, another indicator for the monocyte/macrophage lineage, was gradually increased from P1 to P4 (Supplementary Figure S1D).

We next identified the colitogenic fraction within the P1-P4 subpopulations (Figure 1). At steady state (before DSS challenge), most of the colon $\mathrm{CD} 64^{+}$cells were P3 and P4 (Figure 1a, left panel). In contrast, on day 6 of the DSS challenge, $\sim 60 \%$ of the colon $\mathrm{CD} 64^{+}$cells were $\mathrm{P} 1$ and $\mathrm{P} 2$, and $\sim 36 \%$ were $\mathrm{P} 3$ and $\mathrm{P} 4$ (Figure 1a, middle panel). The numbers of cells in P2 greatly decreased and in P3 also significantly decreased by day 10 after DSS challenge (4 days after ending the DSS treatment) (Figure 1a, right panel, and Figure 1c), at which time the crypt structure had begun to recover (under restoration, Figure 1b). Although each of these subpopulations increased in absolute numbers on day 6 of the DSS challenge, the largest increases were in the $\mathrm{P} 1$ and $\mathrm{P} 2$ populations, and the smallest increase was in the $\mathrm{P} 4$ population (Figure 1a,c). To investigate the functional consequence of each macrophage fraction, we compared the mRNA expression of pro- and antiinflammatory mediators in each subpopulation in steady-state vs. DSS-challenged (day 6) conditions. The expressions of the transcription factor Stat 1 and the proinflammatory mediators Tnf and Nos 2 were enhanced in the $\mathrm{P} 1$ and $\mathrm{P} 2$ populations from DSS-challenged mice (day 6) compared with the same populations from steady-state mice, although the upregulation of Tnf in P1 was not significant $(P=0.082$, Figure 1d). On the other hand, the Il6 and $I l 10$ expressions were unaffected and remained comparable in each subpopulation between the steady-state and DSS-challenged (day 6) mice. Collectively, these results suggested that the colitogenic cells in the P1-P4 subpopulations are the inflammatory monocytes (P1) and inflammatory macrophages (P2).

\section{Requirement of the IFN- $\gamma$-Stat1 axis for colitogenic macrophage development}

Phosphorylated Stat 1 is detected in the monocytic cells from patients with ulcerative colitis but not from those with Crohn's disease. ${ }^{19}$ Given that the Stat 1 expression was greatly enhanced in inflammatory monocytes (P1) and inflammatory macrophages (P2) but not in other populations in the colon (Figure 1d), we next used Stat1-deficient $\left(\right.$ Stat $\left.1^{-1-}\right)$ mice to test whether the transcription factor Stat 1 is essential for the development of colitogenic cells. Notably, on day 6 of the DSS 
a
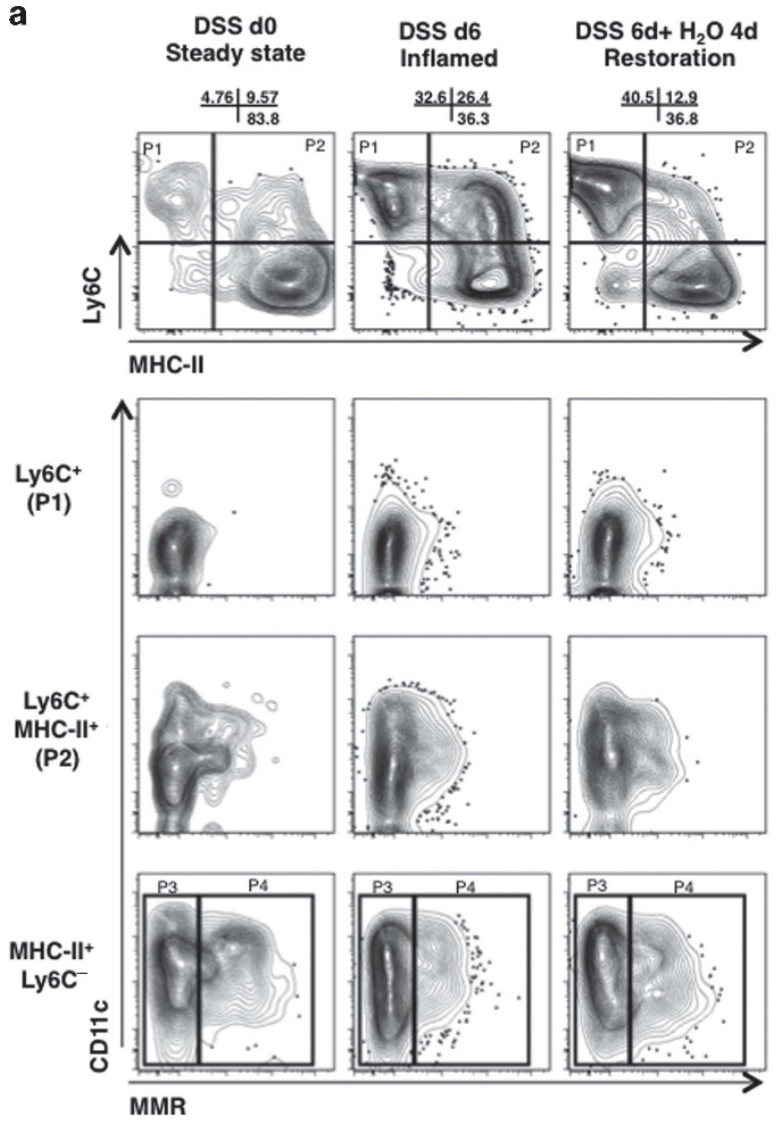

b

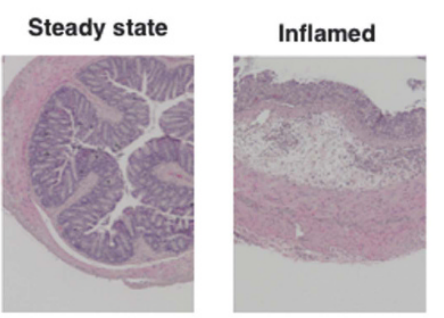

Under restoration

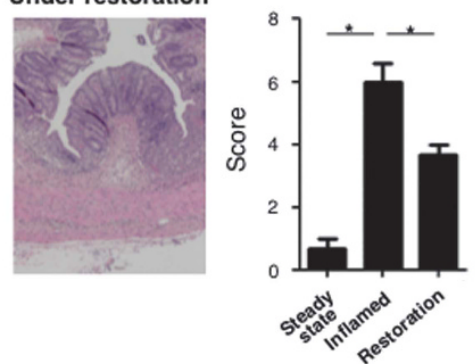

c

Total CD64+ cells

P1

P2

P3

P4
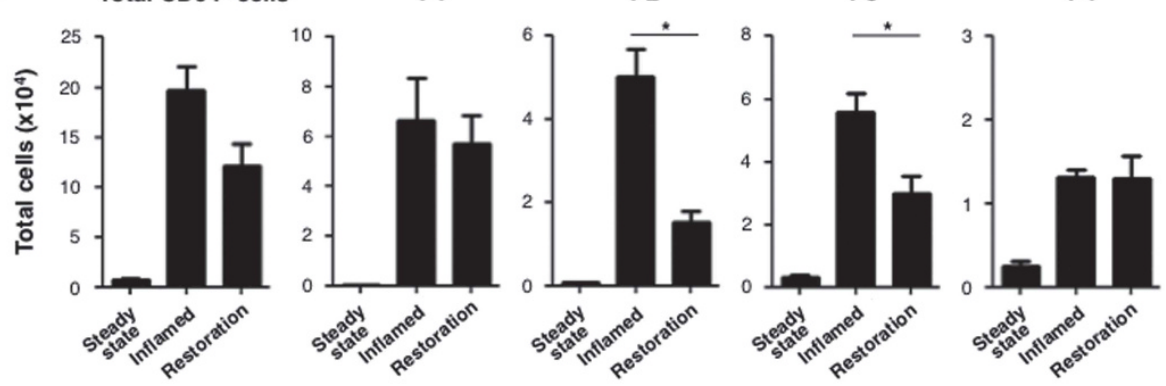

d

Stat1
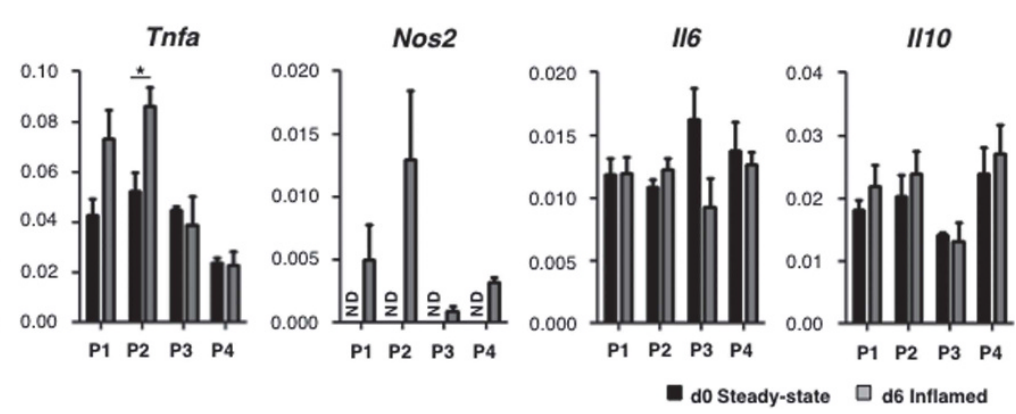

Figure 1 Identification of colitogenic macrophages during colitis development. (a) Flow cytometry analysis of colon lamina propria cells on day (d) 0 (left), 6 (middle), or 10 (right) after beginning a 6-day dextran sodium sulfate (DSS) treatment: Ly6C and MHC-II expression on CD64 ${ }^{+}$cells after gating

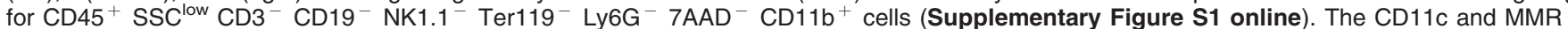
expressions on each population are shown. Numbers indicate the percentage of cells in the P1, P2, and P3 + P4 subsets. (b) Hematoxylin and eosin staining of the distal colon under steady-state, inflamed, and restoration conditions. Original magnification $\times 40$. Histological scores were assigned as described in Methods. (c) Absolute numbers of the indicated subpopulations 0, 6, and 10 days after beginning a 6-day DSS treatment. The data were pooled from three independent animals and are presented as mean \pm SEM ( $n=3$ mice per group). (d) mRNA levels of inflammatory and antiinflammatory mediators in the indicated subpopulations, assayed by qPCR. Data were normalized to Gapdh; the $\Delta \mathrm{CT}$ values (mean \pm s.e.m.) of three independent experiments are shown. ${ }^{\star} P<0.05, \mathrm{ND}$, not detectable. 
challenge, the development of inflammatory macrophages (P2) was significantly impaired in Stat $1^{-1-}$ mice compared with their control littermates (Figure 2a-c). The absence of Stat 1 did not affect the generation of other subpopulations, including the recruitment of inflammatory monocytes (P1), eosinophils, and neutrophils into the inflamed colon or the composition of the $\mathrm{P} 1-\mathrm{P} 4$ subpopulations in the steady state (Figure $\mathbf{2 b}, \mathbf{c}$ and Supplementary Figure S2A-C). As the defective generation of inflammatory macrophages in Stat $1^{-1-}$ mice was not due to the diminished mobilization of inflammatory monocytes into the inflamed colon, these results indicated that Stat1 is important for the optimal generation of inflammatory macrophages from inflammatory monocytes in the inflamed colon.

Stat 1 is activated by proinflammatory cytokines such as IFN$\alpha / \beta$, IFN- $\gamma$, and IL- $6 .{ }^{20}$ As Stat 1 was upregulated but the IL- 6 expression was unaltered in the inflamed colon (Figure 1d), we focused on IFNs. To determine which type of IFN activates Stat1, we used DSS to challenge mice lacking Ifnar 1 (a receptor for IFN- $\alpha / \beta$ ) or IfngrI (a receptor for IFN- $\gamma$ ). Importantly, the generation of inflammatory macrophages (P2) was impaired in Ifngr $1^{-1-}$ mice but not Ifnar $1^{-1-}$ mice (Figure 2d,e and Supplementary Figure S2D). Like the Stat $1^{-1-}$ mice, the Ifngr $^{-1-}$ mice did not show any change in its baseline phenotype (Supplementary Figure S2E,F). In this context, the DSS-challenged mice produced significantly more IFN- $\gamma$ in the inflamed colon and serum than did unchallenged mice (Supplementary Figure 2G). As the distinct composition of the commensal bacteria in Ifngr $1^{-1-}$ mice might affect their susceptibility to DSS-induced colitis, ${ }^{21,22}$ we also confirmed that the development of inflammatory macrophages was impaired in the colon of wild-type (WT) mice treated with XMG1.2, a neutralizing antibody against IFN- $\gamma$ (Supplementary Figure S2H,I). Collectively, these results suggested that the IFN- $\gamma$-dependent activation of Stat1 plays a critical role in generating the colitogenic inflammatory macrophages from inflammatory monocytes.

\section{Milder colitis development in Stat1 ${ }^{-1-}$ mice}

Stat $1^{-1-}$ mice were assessed histologically to determine whether the impaired development of $\mathrm{Ly} 6 \mathrm{C}^{+}$macrophages in these mice ameliorates colitis. On day 6 of the DSS challenge, the colon tended to be longer in Stat $1^{-1-}$ than in control mice (Figure 3a). Compared with control mice, Stat ${ }^{-1-}$ mice showed milder crypt destruction, less goblet-cell loss, and less confluence of inflammatory cell infiltration in the submucosa of the colon, although these parameters were comparable in the two strains before DSS challenge (Figure $3 \mathbf{b}, \mathbf{c}$ ). Thus, the impaired development of inflammatory macrophages correlated well with the milder colitis observed in Stat ${ }^{-1-}$ mice, implying that the Stat1-dependent development of inflammatory macrophages promotes the pathology of colitis.

\section{Maintenance of newly generated inflammatory macrophages by IFN- $\gamma$}

We next examined the role of IFN- $\gamma$ in maintaining inflammatory macrophages in the colon of DSS-challenged mice. Monocytes were isolated from C $x 3 c r 1-g f p$ knock-in mice after 6 days of DSS challenge, and CX3CR1-GFP ${ }^{+}$monocytes were adoptively transferred into DSS-challenged WT recipients (see Methods for details). At $20 \mathrm{~h}$ after the transfer, the inflammatory monocytes had given rise to $\mathrm{MHC}^{-\mathrm{II}^{+}}$inflammatory macrophages. However, $30 \mathrm{~h}$ after transfer, the inflammatory macrophages began to lose their Ly6C expression, and, by $40 \mathrm{~h}$ after transfer, had further converted into migrant macrophages that did not express MMR. We also transferred CX3CR1-GFP \pm monocytes into noninflamed WT recipients. At $40 \mathrm{~h}$ after the transfer, when $\mathrm{MHC}^{-\mathrm{II}^{+}} \mathrm{Ly} 6 \mathrm{C}^{+}$ macrophages were present in the inflamed host, most of the monocytes became $\mathrm{MHC}-\mathrm{II}^{+} \mathrm{Ly}_{6 \mathrm{C}^{-}}$macrophages expressing higher level of CX3CR1 (CX3CR1 $1^{\text {high }}$ ), one of the representative markers for resident macrophages, in the noninflamed host (Supplementary Figure S3A, B). This observation was consistent with previous reports demonstrating that it is difficult for inflammatory monocytes to differentiate into resident macrophages in the inflammatory setting. ${ }^{12,18}$

To explore the role of IFN- $\gamma$, a 1:1 mixture of CX3CR1$\mathrm{GFP}^{+}$Ifngr ${ }^{-1-}$ monocytes (CD45.2) and WT CX3CR1$\mathrm{GFP}^{+}$monocytes (CD45.1) was transferred into WT recipients (CD45.2) on day 4 of the DSS challenge (Figure 4). At $30 \mathrm{~h}$ after the transfer, $\sim 80 \%$ of the inflammatory macrophages (P2) were of WT monocyte origin. In contrast, the inflammatory monocytes (P1) were composed of a 1:1 mixture of Ifngr $1^{-1-}$ and WT monocytes, indicating that intrinsic IFN $-\gamma$ signaling is required to maintain newly generated inflammatory macrophages, rather than the migration of monocytes into the inflamed colon. Supporting this notion, the proportion of Ifngr $1^{-1-}$ monocyte-derived migrant/resident macrophages (P3 and P4) was higher than that of Ifngr $1^{-1-}$ monocytederived inflammatory macrophages (P2). Collectively, these results indicated that intrinsic IFN- $\gamma$ signaling is important for the maintenance of inflammatory macrophages in the inflamed colon.

It was recently suggested that IFN- $\gamma$ from natural killer (NK) cells is crucial for educating monocytes to acquire an antiinflammatory function in an infection-induced colitis model. ${ }^{23}$ The action of IFN- $\gamma$ can be evaluated by the upregulation of Sca-1, a representative IFN-inducible protein, in monocytes and their $\mathrm{c}-\mathrm{MoP}$ progenitors in the bone marrow. However, in our experimental setting (the DSS-induced colitis model), Sca-1 was not upregulated on bone marrow monocytes (Supplementary Figure S4). Collectively, these results suggest that IFN- $\gamma$ helps to maintain the colitogenic macrophages generated from monocytes in the inflamed colon, rather than educating monocytes in the bone marrow that will later be recruited to the colon. In our experimental setting, the cell types that produce IFN- $\gamma$ in the inflamed colon have not been identified. In general, T helper type 1 cells and NK cells are major producers of IFN- $\gamma$. However, depleting these cell types by administrating GK1.5 and PK136 monoclonal antibodies did not affect the development of colitogenic macrophages (data not shown), and various cell types produced IFN- $\gamma$, including CD4, CD8, $\mathrm{CD}^{+}{ }^{+} \mathrm{NK} 1.1^{+}$NKT-like cells, NKp46 ${ }^{+}$ILC1-like cells, and 


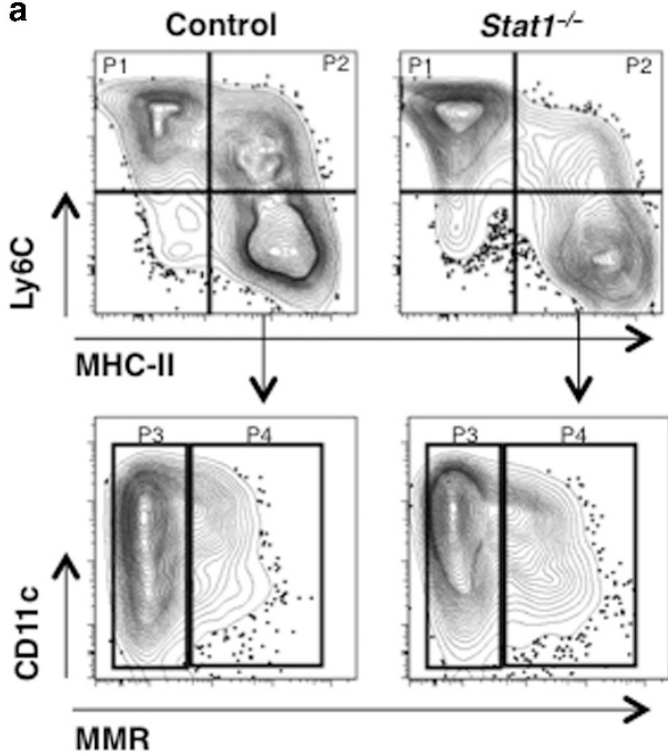

C

Total $\mathrm{CD} 4^{+}$cells

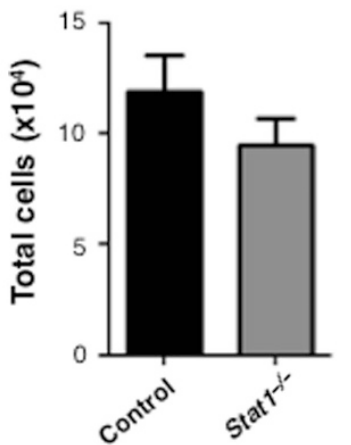

P1

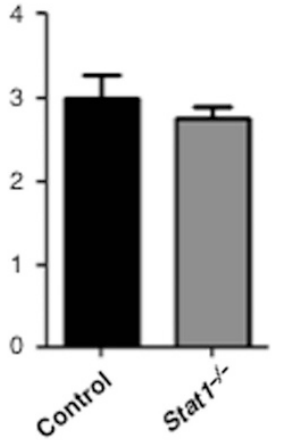

P2

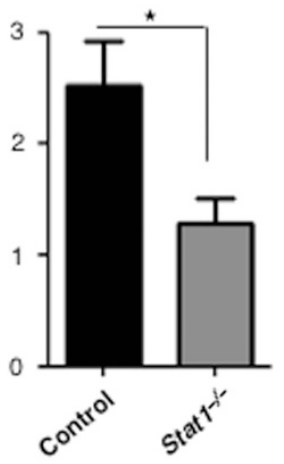

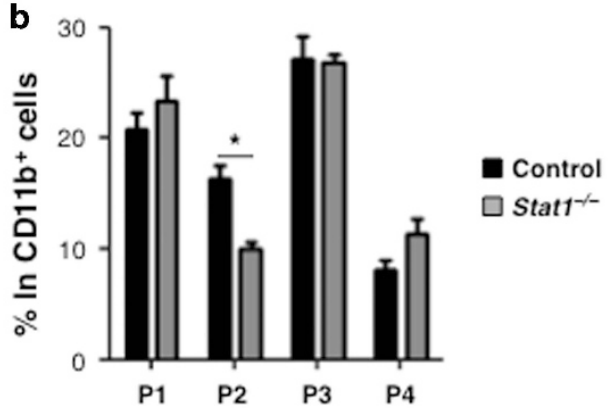

P3

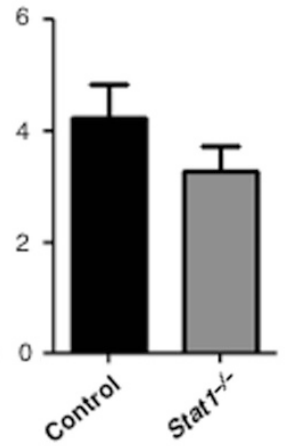

P4

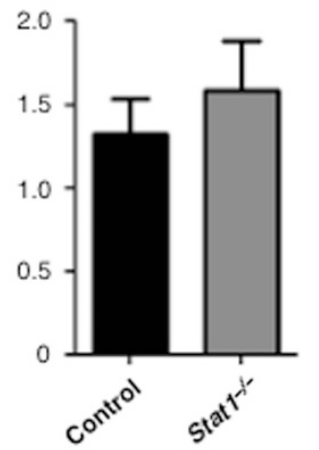

d
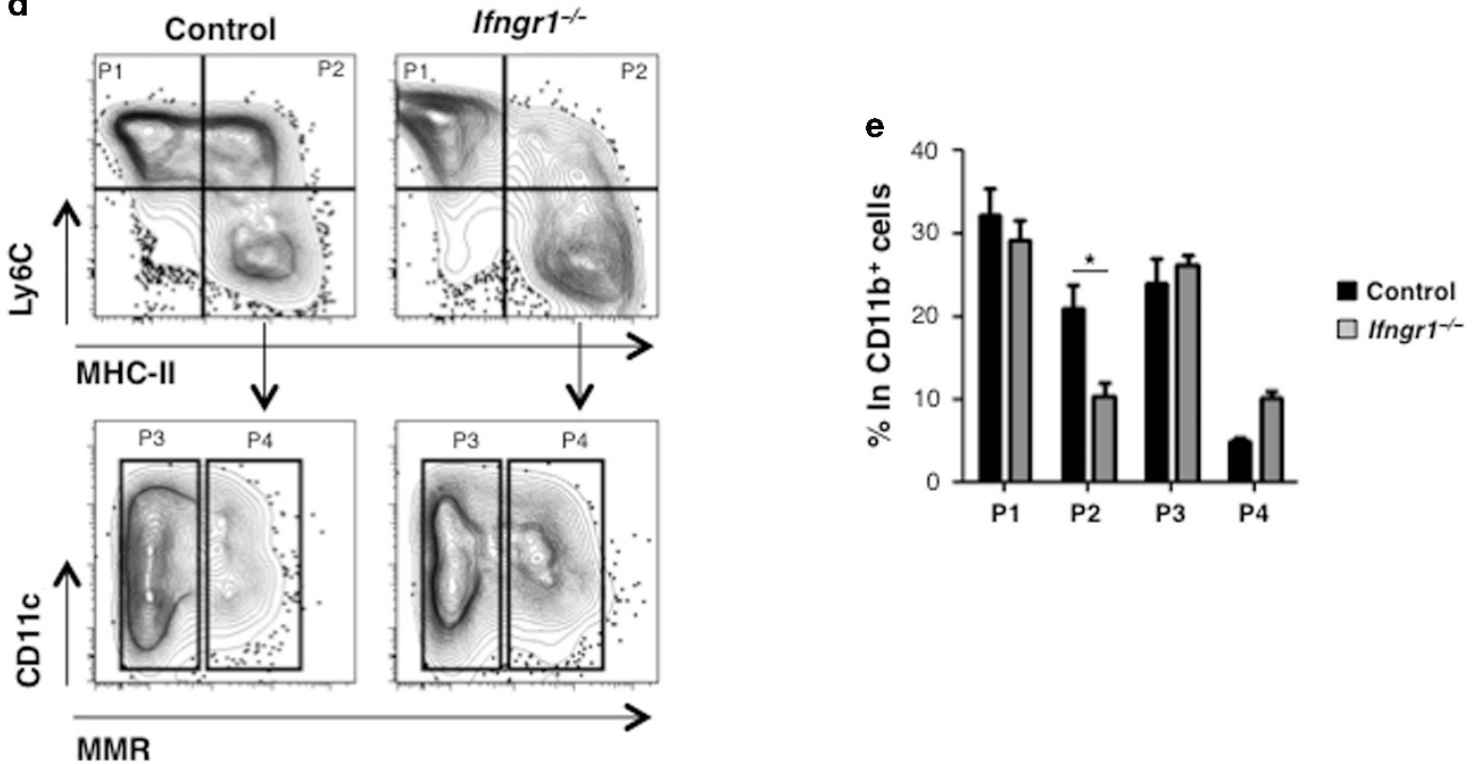

Figure 2 The transcription factor signal transducer and activator of transcription 1 (STAT1) is required for the development of colitogenic macrophages. $(\mathbf{a}-\mathbf{c})$ Flow cytometry analysis $(\mathbf{a})$, percentages $(\mathbf{b})$, and absolute numbers $(\mathbf{c})$ of the indicated subpopulations obtained from the colon of Stat $1^{+\prime}-$ control littermates or Stat $1^{-1-}$ mice after 6 days of dextran sodium sulfate (DSS) treatment. The data were pooled from three independent experiments and are presented as mean \pm s.e.m. ( $n=8-9$ mice per group). (d, e) Flow cytometry analysis (d) and percentages (e) of the indicated subpopulations obtained from the colon of Ifngr $1^{+/-}$control littermates or Ifngr $1^{-1-}$ mice after 6 days of DSS treatment. The data were pooled from three independent mice and are presented as mean \pm s.e.m. ${ }^{*} P<0.05$. 
a

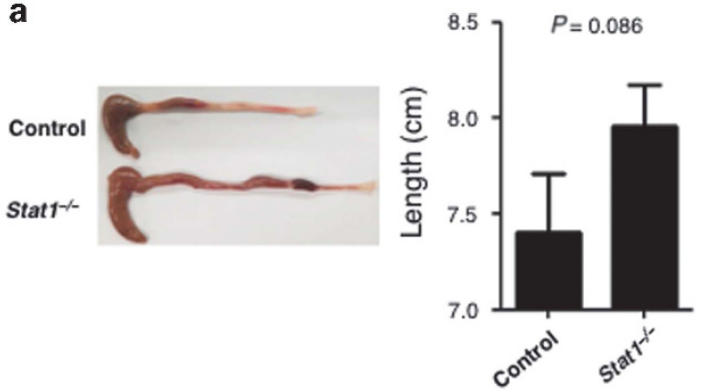

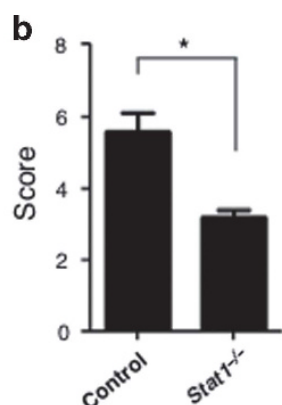
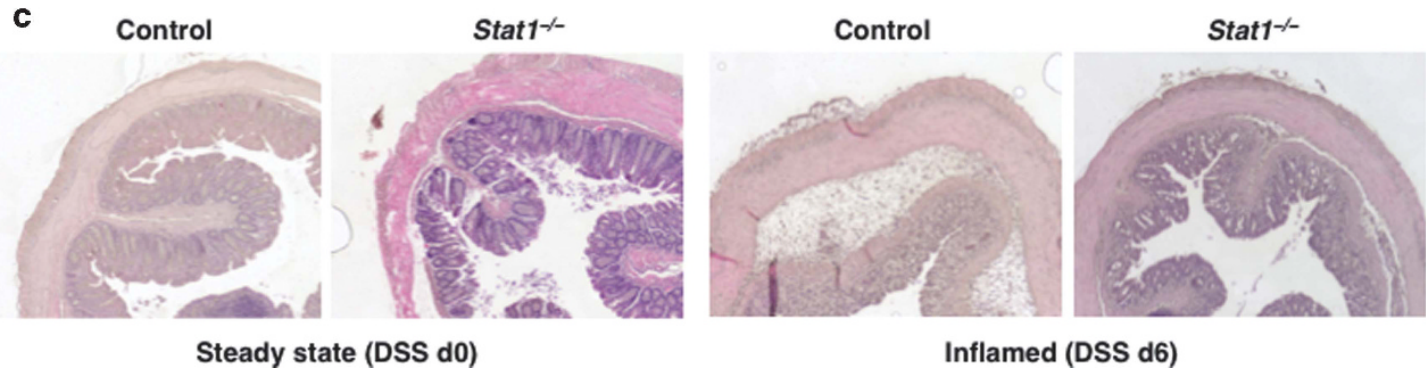

Inflamed (DSS d6)

Figure 3 Effect of the low colitogenic macrophage counts in Stat1 ${ }^{-1}$ mice on colitis development. (a) Colon length after 6 days of dextran sodium sulfate (DSS) challenge in Stat $1^{-1-}$ mice and control littermates ( $n=5$ mice/group). (b, c) Hematoxylin and eosin staining of the distal colon from Stat $1^{-1}$ and control mice. Original magnification $\times 40$. Histological scores were assigned as described in the Methods. The data were pooled from three independent experiments and are presented as mean \pm s.e.m. ( $n=5$ mice per group). ${ }^{\star} P<0.05$.

P1

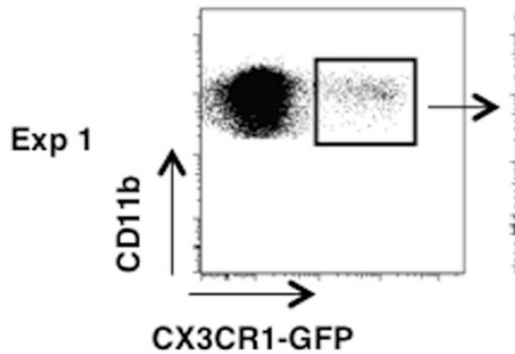

CX3CR1-GFP
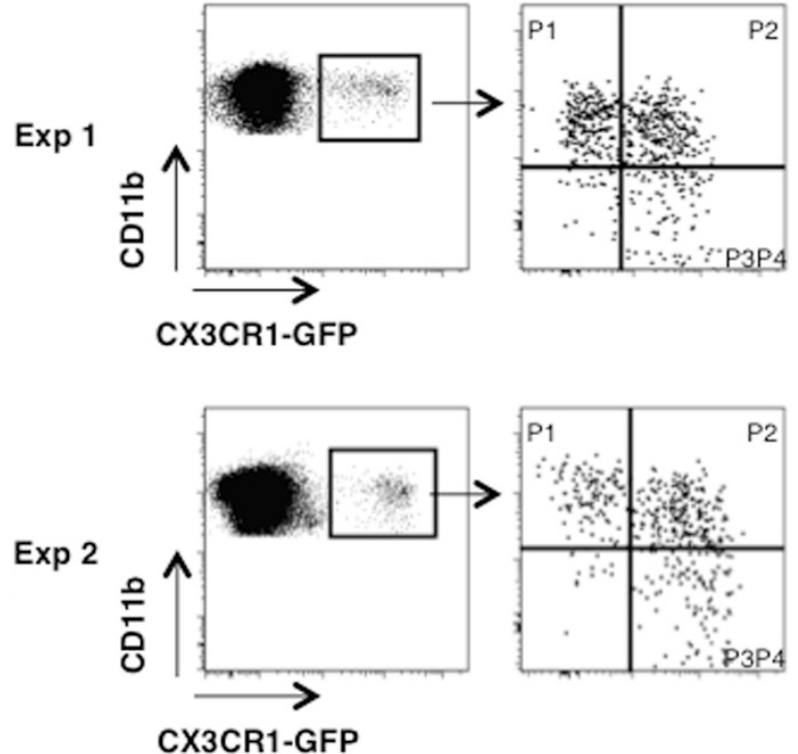

4
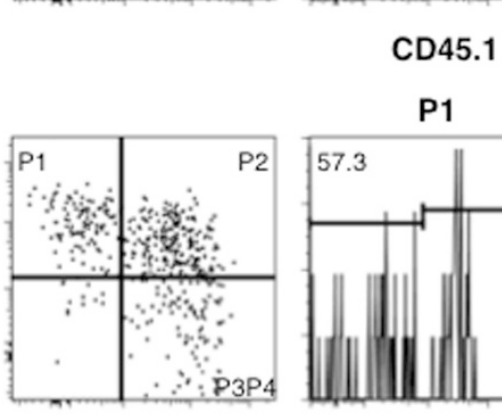

CX3CR1-GFP
P1

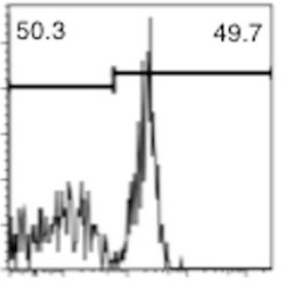

D45.1

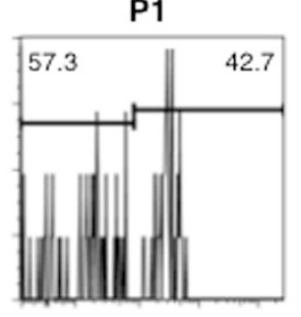

CD45.1
P2

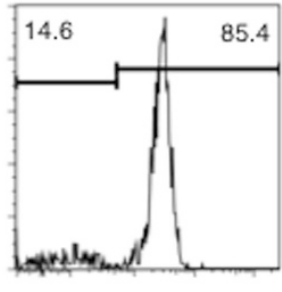

CD45.1

P2

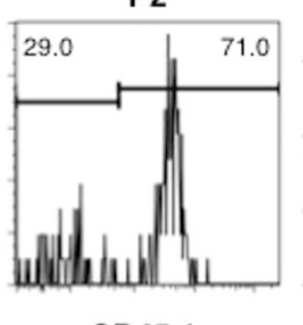

CD45.1
P3P4

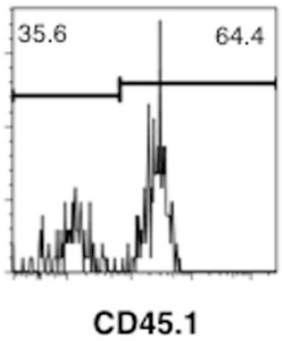

P3P4

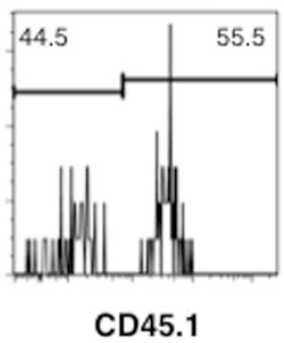

Figure 4 Interferon- $\gamma($ IFN- $\gamma$ ) is required for the generation of colitogenic macrophages. Flow cytometry analysis of wild-type (WT) recipient mice $30 \mathrm{~h}$ after adoptive transfer of CX3CR $1^{+}$monocytes that were composed of $10^{6}$ WT monocytes (CD45.1) and $10^{6}$ Ifngr $1^{-1-}$ monocytes (CD45.2). The adoptive transfer was performed 4 days after dextran sodium sulfate (DSS) treatment. Results of two independent experiments are shown.

$\mathrm{CD} 3{ }^{-} \mathrm{CD} 4{ }^{+}$LTi-like cells (Supplementary Figure S5), as previously reported. ${ }^{24}$

\section{Increased colitogenic-mediator gene transcription by IFN- $\gamma$ through histone acetylation}

We next sought to elucidate the mechanisms by which IFN- $\gamma$ regulates TNF and iNOS in the monocyte/macrophage lineage in the inflamed colon. Commensal bacteria and their products, including Toll-like receptor ligands, trigger inflammatory responses by macrophages, and these inflammatory responses are associated with IBD pathology. Specifically deleting MyD88 in macrophages attenuates the severity of colitis in IL-10deficient mice. ${ }^{25}$ In this context, effective Toll-like receptor signaling is necessary for chromatin remodeling, including acetylation, to transcribe DNA. ${ }^{26-28}$ Thus, we hypothesized that IFN- $\gamma$ regulates the gene expression of inflammatory mediators through histone acetylation to promote colitogenicity. To test this hypothesis, we assayed the mRNA levels of 
inflammatory mediators in the colitogenic cells $(\mathrm{P} 1+\mathrm{P} 2)$ sorted from the colon of steady-state mice (steady state) and the inflamed colon of mice treated with either a control antibody (isotype) or the neutralizing anti-IFN- $\gamma$ antibody, XMG1.2 (Figure 5a). Strikingly, the mRNA levels of colitogenic mediators such as Tnf and Nos2 were dramatically increased upon colitis induction and decreased by treatment with the neutralizing anti-IFN- $\gamma$ antibody, whereas Il6 was not affected. Anti-IFN- $\gamma$ treatment also reduced the Stat1 expression. Similar results were obtained using Stat $1^{-1-}$ mice (Supplementary Figure S6), suggesting that the IFN- $\gamma$-Stat1 axis is essential for the induction of colitogenic mediators from the colitogenic cells in the inflamed colon.

To test whether IFN- $\gamma$ enhances the transcription of colitismediating genes via histone acetylation, we performed chromatin immunoprecipitation (ChIP) assays using antibodies against acetyl-histone 4. ChIP-quantitative PCR revealed that the acetylation was elevated at the promoter regions for Tnf and Nos 2 but not Il6 in the colitogenic cells isolated from the inflamed colon of DSS-treated mice (Figure 5b). Remarkably, the histone acetylation status was canceled by IFN- $\gamma$ neutralization in vivo (Figure $5 \mathbf{b}$ ), showing that IFN- $\gamma$ signaling is essential for histone acetylation in the promoter region of colitis-mediating genes. The transcription factor p65, also known as RELA, is involved in forming nuclear factor $(\mathrm{NF})-\kappa \mathrm{B}$ heterodimers and activating inflammatory cytokine genes. ${ }^{29}$ Thus, we investigated the p65 activation (Figure 5c). Phosphorylated p65 was increased upon DSS challenge and remained elevated despite treatment with a neutralizing anti-IFN- $\gamma$ antibody, suggesting that IFN- $\gamma$ is dispensable for $\mathrm{p} 65$ activation. In line with these results, IFN- $\gamma$ minimally affected the Toll-like receptor signaling in macrophages upon lipopolysaccharide stimulation in vitro. ${ }^{30}$ Collectively, our results show that IFN- $\gamma$ induces genes that encode colitis mediators via histone modification, causing macrophages in the inflamed colon to produce these inflammatory mediators.

\section{DISCUSSION}

In this study, we showed that $\mathrm{CD}^{+} 4^{+}$cells in the inflamed colon are divided into four major subpopulations, namely inflammatory monocytes, inflammatory macrophages, migrant macrophages, and resident macrophages. Among a

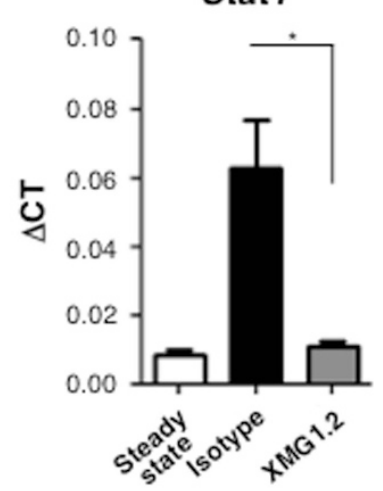

Tnfa

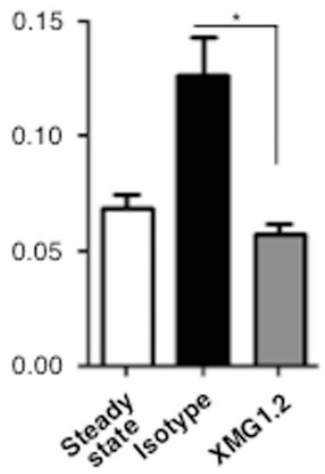

Nos2

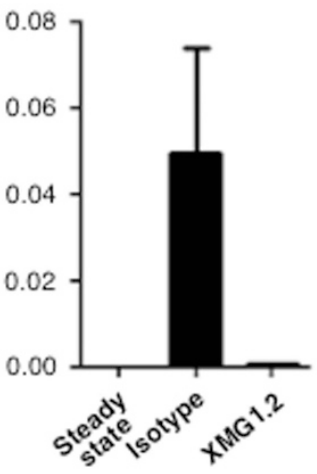

116

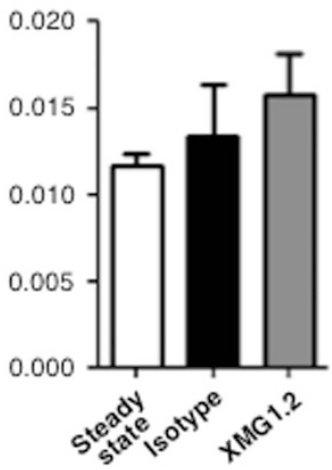

b

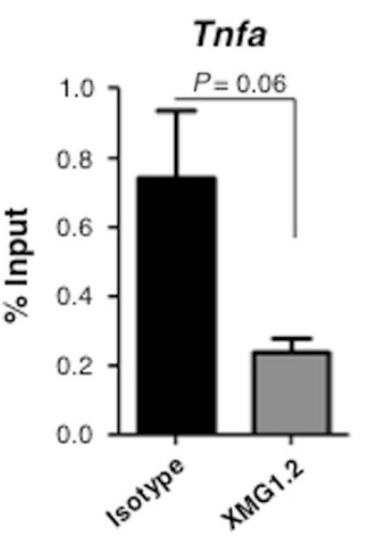

Nos2

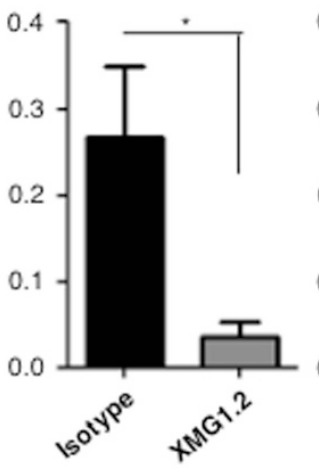

116

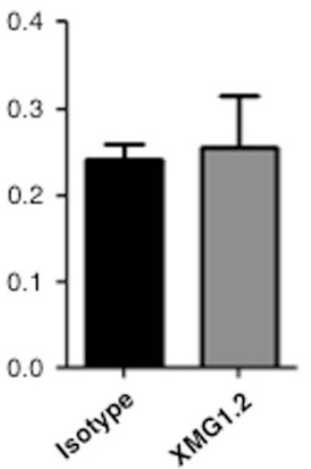

c
Total NF-KB p65
PhosphoNF-кB p65

Figure 5 Interferon- $\gamma($ IFN- $\gamma$ ) upregulated colitogenic genes via enhanced acetylation. (a) mRNA of the indicated genes in the sorted P1 + P2 populations from steady-state mice and colitic mice treated with either isotype control or XMG1.2, measured by quantitative PCR (qPCR) and normalized to the Gapdh mRNA. The $\triangle \mathrm{CT}$ values (mean \pm s.e.m.) of three independent experiments are shown. ${ }^{\star} P<0.05$. (b) Samples were analyzed by chromatin immunoprecipitation (ChIP) using antibodies against acetyl histone 4. Purified DNA was analyzed using qPCR primers specific to the promoter of the indicated genes. Normalized results are shown as a percentage of input. ${ }^{\star} P<0.05$. (c) Total and phosphorylated nuclear factor (NF- $\mathrm{B}$ ) p65 in the colitogenic P1 + P2 populations analyzed by enzyme-linked immunosorbent assay (ELISA). Data are combined from two independent experiments. Error bars represent mean \pm s.e.m. 
these subpopulations, we identified the former two as highly colitogenic populations that produce colitis-associated inflammatory mediators such as TNF and iNOS. In this context, the generation of inflammatory macrophages from inflammatory monocytes depended on IFN- $\gamma-$ Stat1 signaling. This mechanism may account for the previous observation that IFN $-\gamma^{-1-}$ mice are resistant to DSS colitis. ${ }^{31}$

Our findings also showed that IFN- $\gamma$ instructs the development of the colitogenic monocyte/macrophage lineage in response to DSS challenge in vivo by epigenetically controlling the transcription of inflammatory mediators via the transcription factor STAT1. Supporting these findings, an in vitro study using human macrophages revealed that IFN- $\gamma$ induces STAT1 and IRF1 occupancy and histone acetylation at the promoter region of the Tnf, Il6, and $I l 12 b$ genes, ${ }^{30}$ thus increasing the transcription of these cytokines in response to lipopolysaccharide stimulation. We observed defects in the development of colitogenic macrophages in both Ifngr ${ }^{-1-}$ and Stat $1^{-1-}$ mice, and showed that the IFN- $\gamma$-Stat1 axis is essential for histone acetylation at the loci of colitogenic mediators such as Tnf and Nos2 in the colonic monocyte/macrophage lineage of DSS-injected mice. These results suggested that the IFN- $\gamma-$ Stat1 pathway affects both the differentiation of inflammatory monocytes into inflammatory macrophages and the transcription of inflammatory genes in these cells. Deacetylase inhibitor trichostatin A or butyrate suppresses the transcription of inflammatory genes such as Nos 2 and Il6 in colonic macrophages in the steady state. However, in vivo butyrate treatment did not affect the severity of DSS-induced colitis, ${ }^{32}$ implying that different mechanisms regulate gene expression in the steady state vs. the inflammatory environment. Based on existing literature and our present findings, inhibiting acetyl transferase rather than deacetylase might more effectively improve IBD symptoms by regulating the colitogenic monocyte/macrophage lineage.

We note that the reduction in P2 cells was relatively modest in the inflamed colon of Ifngr $1^{-1-}$ and Stat $1^{-1-}$ mice. However, the susceptibility of colitis is affected not only by the frequencies but also by the colitogenic properties of the $\mathrm{P} 1$ and P2 cells. Indeed, the expression levels of Tnfa and Nos2 were significantly reduced in these cells in WT mice treated with XMG1.2 (an anti-IFN $\gamma$ neutralizing antibody) and in Stat $^{-1-}$ mice (Figure 5a and Supplementary Figure S6) that would substantially contribute to the improvement of colitis.

Overall, our findings present the education mechanism for colitogenic macrophages during their local differentiation from monocytes. The origin and fate of colon macrophages in the steady state and in an inflammatory context are well studied, ${ }^{12,18}$ but the mechanism (including the master transcriptional regulator) by which macrophages acquire colitogenicity in vivo has not been fully clarified. Addressing this issue, our study revealed that the IFN- $\gamma-$ Stat 1 pathway plays a critical role in generating colitogenic macrophages by promoting transcription of the Tnf and Nos2 genes via histone acetylation. In addition to these epigenetic changes, a recent study showed that IFN- $\gamma$ selectively modulates macrophage translatomes to promote inflammation, to further reprogram metabolic pathways, and to regulate protein synthesis after Toll-like receptor-2 stimulation in vitro. ${ }^{33}$ Epigenetic reprogramming, signaling pathways, and metabolic processes are all known to mediate macrophage training. ${ }^{34,35}$ By identifying the colitogenic monocyte and macrophage subsets and their induction machinery, our study may provide new therapeutic targets for IBD and colon cancer.

\section{METHODS}

\section{Mice and DSS induction of colitis}

We obtained C57BL/6 (B6) mice from SLC Japan (Shizuoka, Japan), B6.SJL-ptprca (B6.SJL) mice congenic at the CD45 locus $\left(\mathrm{CD} 45.1^{+} \mathrm{CD} 45.2^{-}\right)$from Taconic (Rensselaer, NY), and B6.Stat $1^{-1-}$, B6.Ifngr1 ${ }^{-1-}$, and Cx3cr1-gfp mice from Jackson Laboratory (Bar Harbor, ME). Mice were kept 5-6/cage under specific-pathogen-free conditions using the Micro-VENT system (Allentown, PA) and were maintained on sterile chow. All of the animal experiments were approved by the Institutional Animal Care Committee of Tokyo Medical and Dental University. Mice were given 2\% (wt/vol) DSS (MP Biochemicals, Santa Ana, CA) in the drinking water for 6-7 days. In some experiments, WT hosts received a neutralizing anti-IFN- $\gamma$ antibody ( $400 \mu \mathrm{g} /$ per mouse) (Bio X cells, West Lebanon, MA).

\section{Intestinal cell preparation and flow cytometry}

Colonic lamina propria cells were isolated as previously described. ${ }^{13}$ The colon was opened, washed with phosphate-buffered saline (PBS), cut into segments, and shaken in PBS containing $5 \mathrm{~mm}$ EDTA. The tissue was thoroughly washed in PBS to remove residual epithelial cells, cut into tiny pieces with scissors, and digested by shaking in PBS containing $0.1 \%$ type IV collagenase (Sigma-Aldrich, St Louis, MO) for $30 \mathrm{~min}$ at $37^{\circ} \mathrm{C}$. The cells were filtered through a $100 \mu \mathrm{m}$ cell strainer, washed in $1 \%$ fetal calf serum-PBS, and stained with a combination of anti-CD11c (N418), anti-CD11b (M1/70), antiI-A/I-E (M5/114.15.2), anti-CD64 (X54-5/7.1), anti-CD45 (30F11), anti-CD45.1 (A20), anti-Ly6C (HK1.4), anti-Ly6G (1A8), anti-CD3 (145-2C11), anti-CD19 (6D5), anti-NK1.1 (PK136), anti-Ter119 (TER119), anti-CCR2 (475301), and 7-aminoactinomycin D after blocking Fc receptors. Antibodies were obtained from Biolegend (San Diego, CA), BD Pharmingen (San Diego, CA), or R\&D System (Minneapolis, MN). Flow cytometry and sorting was performed on a FACSAria III (BD Biosciences, San Diego, CA). FlowJo software (Tree Star, Ashland, OR) was used for data analysis.

\section{Adoptive transfer of bone marrow monocytes}

Bone marrow was flushed from the tibias and femurs of donor Cx3cr1-gfp knock-in mice that had been challenged with DSS for 6 days to activate and expand monocytes. After lysing red blood cells with ACK lysing buffer, the cells were stained with phycoerythrin/Cy5- or Alexa-647-conjugated antibodies against CD3, CD19, NK1.1, Ter119, I-A/I-E, B220, and Ly6G. Lineage-marker-positive cells were depleted with anti-Cy5/Alexa-647 microbeads and an AutoMACSpro 
separation system (Miltenyi Biotec, Bergisch Gladbach, Germany) according to the manufacturers' instructions. $\mathrm{C} \times 3 \mathrm{cr} 1^{\text {int }}$, ${\mathrm{c}-\mathrm{kit}^{-}, \mathrm{CD} 11 \mathrm{c}^{-} \text {, and SSC }}^{\text {low }}$ monocytes were then sorted with a FACSAria III. The purified monocytes were transferred intravenously into the tail vein of WT hosts (CD45.1 CD $45.2^{+}$) 4 days after the start of DSS treatment. After transferring, the host colons were analyzed at various time points.

\section{Cytokine measurement in colonic tissue}

Tissue from the distal colon was cut into $1 \mathrm{~cm}$ segments, homogenized with scissors, and suspended in $500 \mu \mathrm{l} \mathrm{Hanks'}$ balanced salt solution containing $0.1 \%$ Triton X-100 and protease and phosphatase inhibitors. Supernatants were collected by centrifugation (10,000 r.p.m., $5 \mathrm{~min}$ ) and assessed for IFN- $\gamma$ (eBioscience, San Diego, CA).

\section{NF-кB p65 measurement}

Total and phosphorylated NF- $\kappa \mathrm{B}$ p 65 were assayed by enzymelinked immunosorbent assay (ELISA) using the InstantOne ELISA Kit (eBioscience).

\section{Histological analysis}

Tissue harvested from the distal colon was cut into segments, fixed with 10\% Formaldehyde Neutral Buffer Solution (Nacalai, Kyoto, Japan) overnight, embedded in paraffin, sectioned at $5 \mu \mathrm{m}$, and stained with hematoxylin and eosin. Histological scores were evaluated as described previously. ${ }^{36}$ The severity of inflammation was graded from 0 to 3 as follows: 0 , rare inflammatory cells in the lamina propria; 1, increased inflammatory cells in the lamina propria; 2, confluent inflammatory cells extending into the submucosa; and 3, the transmural extension of inflammatory cell infiltrate. Damage was graded from 0 to 4 as follows: 0 , none; 1 , loss of the basal $1 / 3$ of the crypt; 2 , loss of the basal $2 / 3$ of the crypt; 3 , loss of the entire crypt but with intact epithelial cells; and 4; loss of the entire crypt and the surface epithelial cells.

\section{Quantitative reverse transcription-PCR}

To measure mRNA expression, total RNA was extracted from sorted macrophages using the RNeasy Mini Kit (Qiagen, Hilden, Germany), and complementary DNA was synthesized using random hexamers and SuperScript III Reverse Transcriptase (Life Technologies, Carlsbad, CA) according to the manufacturer's instructions. Quantitative PCR was performed with a LightCycler 480 Sybr Green I (Roche, Penzbarg, Germany). Primer pairs for each sequence are listed in Supplementary Table S1. Relative expression was normalized to Gapdh. Data are represented as the fold induction over the day-0 total for $\mathrm{CD}^{+} 4^{+} \mathrm{MHC}-\mathrm{II}^{+}$ macrophages.

\section{ChIP assay}

ChIP assays were performed as described previously ${ }^{37}$ with minor modifications: $10^{5}$ colon macrophages were fixed with $1 \%$ formaldehyde for 10 min to obtain crosslinked chromatins. Cells were washed with cold PBS, solubilized with SDS lysis buffer containing protease inhibitors, and sonicated to obtain
DNA fragments. After centrifuging, the supernatants were diluted 10-fold in ChIP dilution buffer and immunoprecipitated with antibodies against acetyl histone 4 (Merck Millipore, Darmstadt, Germany) and with salmon sperm DNA/proteinase A-agarose (Merck Millipore). The immunoprecipitated chromatins were washed, eluted, and incubated at $65^{\circ} \mathrm{C}$ to reverse the crosslinks. After treatment with proteinase $\mathrm{K}$, the recovered DNA was purified with ChIP DNA Clean \& Concentrator (Zymo Research, Irvine, CA), and analyzed by qPCR using gene-specific primers (Supplementary Table S2).

\section{Statistical analysis}

Normally distributed, continuous variables were compared by two-tailed unpaired $t$-tests and one-way analysis of variance followed by Tukey's post comparison, using Prism software.

SUPPLEMENTARY MATERIAL is linked to the online version of the paper at http://www.nature.com/mi

\section{ACKNOWLEDGMENTS}

We thank H. Kamioka for secretarial support and A. Hosono and M. Tsuda for technical support. This work was supported by the Tuchiya Memorial Foundation (to Y.N.), a Grant-in-Aid for Scientific Research (A) from the Ministry of Education, Culture, Sports, Science and Technology (MEXT) of Japan (to T.O.), and the Joint Usage/Research Program of the Medical Research Institute, Tokyo Medical and Dental University (to K.T.).

\section{AUTHOR CONTRIBUTIONS}

Y.N. designed and conducted the experiments, T.S. and K.T. assisted with some experiments, and Y.N. and T.O. analyzed data and prepared the manuscript.

\section{DISCLOSURE}

The authors declared no conflict of interest.

(c) 2018 Society for Mucosal Immunology

\section{REFERENCES}

1. Okabe, Y. \& Medzhitov, R. Tissue biology perspective on macrophages. Nat. Immunol. 17, 9-17 (2016).

2. Epelman, S., Lavine, K.J. \& Randolph, G.J. Origin and functions of tissue macrophages. Immunity 41, 21-35 (2014).

3. Ginhoux, F. \& Jung, S. Monocytes and macrophages: developmental pathways and tissue homeostasis. Nat. Rev. Immunol. 14, 392-404 (2014).

4. Ginhoux, F., Schultze, J.L., Murray, P.J., Ochando, J. \& Biswas, S.K. New insights into the multidimensional concept of macrophage ontogeny, activation and function. Nat. Immunol. 17, 34-40 (2016).

5. Amit, I., Winter, D.R. \& Jung, S. The role of the local environment and epigenetics in shaping macrophage identity and their effect on tissue homeostasis. Nat. Immunol. 1, 18-25 (2016).

6. Butovsky, O. et al. Identification of a unique TGF- $\beta$-dependent molecular and functional signature in microglia. Nat. Neurosci. 17, 131-143 (2014).

7. Haldar, M. et al. Heme-mediated SPI-C induction promotes monocyte differentiation into iron-recycling macrophages. Cell 156, 1223-1234 (2014).

8. Okabe, Y. \& Medzhitov, R. Tissue-specific signals control reversible program of localization and functional polarization of macrophages. Cell 157, 832-844 (2014).

9. Bain, C.C. et al. Constant replenishment from circulating monocytes maintains the macrophage pool in the intestine of adult mice. Nat. Immunol. 15, 929-937 (2014).

10. Tamoutounour, S. et al. CD64 distinguishes macrophages from dendritic cells in the gut and reveals the Th1-inducing role of mesenteric lymph node macrophages during colitis. Eur. J. Immunol. 42, 3150-3166 (2012). 
11. Zigmond, E. et al. Macrophage-restricted interleukin-10 receptor deficiency, but not IL-10 deficiency, causes severe spontaneous colitis. Immunity 40, 720-733 (2014).

12. Zigmond, E. et al. Ly6C hi monocytes in the inflamed colon give rise to proinflammatory effector cells and migratory antigen-presenting cells. Immunity 37, 1076-1090 (2012).

13. Nakanishi, Y., Sato, T. \& Ohteki, T. Commensal Gram-positive bacteria initiates colitis by inducing monocyte/macrophage mobilization. Mucosal Immunol. 8, 152-160 (2015).

14. Asano, K. et al. Intestinal CD169(+) macrophages initiate mucosal inflammation by secreting CCL8 that recruits inflammatory monocytes. Nat. Commun. 6, 7802 (2015).

15. Goretsky, T. et al. p53 mediates TNF-induced epithelial cell apoptosis in IBD. Am. J. Pathol. 181, 1306-1315 (2012).

16. Rivollier, A., He, J., Kole, A., Valatas, V. \& Kelsall, B.L. Inflammation switches the differentiation program of Ly6Chi monocytes from antiinflammatory macrophages to inflammatory dendritic cells in the colon. J. Exp. Med. 209, 139-155 (2012).

17. Bain, C.C. \& Mowat, A.M. Macrophages in intestinal homeostasis and inflammation. Immunol. Rev. 260, 102-117 (2014).

18. Bain, C.C. et al. Resident and proinflammatory macrophages in the colon represent alternative context-dependent fates of the same Ly6Chi monocyte precursors. Mucosal. Immunol. 6, 498-510 (2013).

19. Schreiber, S. et al. Activation of signal transducer and activator of transcription (STAT) 1 in human chronic inflammatory bowel disease. Gut 51, 379-385 (2002).

20. Wilson, H.M. SOCS proteins in macrophage polarization and function. Front. Immunol. 5, 357 (2014).

21. Elinav, E. et al. NLRP6 inflammasome regulates colonic microbial ecology and risk for colitis. Cell 145, 745-757 (2011).

22. Levy, M. et al. Microbiota-modulated metabolites shape the intestinal microenvironment by regulating NLRP6 inflammasome signaling. Cell 163, 1428-1443 (2015).

23. Askenase, M.H. et al. Bone-marrow-resident NK cells prime monocytes for regulatory function during infection. Immunity 42, 1130-1142 (2015).
24. Nava, P. et al. Interferon-gamma regulates intestinal epithelial homeostasis through converging beta-catenin signaling pathways. Immunity 32 , 392402 (2010).

25. Hoshi, N. etal. MyD88 signalling in colonic mononuclear phagocytes drives colitis in IL-10-deficient mice. Nat. Commun. 3, 1120 (2012).

26. Foster, S.L. \& Medzhitov, R. Gene-specific control of the TLR-induced inflammatory response. Clin. Immunol. 130, 7-15 (2009).

27. Medzhitov, R. \& Horng, T. Transcriptional control of the inflammatory response. Nat. Rev. Immunol. 9, 692-703 (2009).

28. Ivashkiv, L.B. Epigenetic regulation of macrophage polarization and function. Trends Immunol. 34, 216-223 (2013).

29. Hargreaves, D.C., Horng, T. \& Medzhitov, R. Control of inducible gene expression by signal-dependent transcriptional elongation. Ce/l 138, 129145 (2009).

30. Qiao, Y. et al. Synergistic activation of inflammatory cytokine genes by interferon- $\gamma$-induced chromatin remodeling and toll-like receptor signaling. Immunity 39, 454-469 (2013).

31. Ito, R. et al. Interferon-gamma is causatively involved in experimental inflammatory bowel disease in mice. Clin. Exp. Immunol. 146, 330-338 (2006).

32. Chang, P.V., Hao, L., Offermanns, S. \& Medzhitov, R. The microbial metabolite butyrate regulates intestinal macrophage function via histone deacetylase inhibition. Proc. Natl. Acad. Sci. USA 111, 2247-2252 (2014).

33. Su, $X$. et al. Interferon- $\gamma$ regulates cellular metabolism and mRNA translation to potentiate macrophage activation. Nat. Immunol. 16, 838-849 (2015).

34. Cheng, S.C. et al. mTOR- and HIF-1 $1 \alpha$-mediated aerobic glycolysis as metabolic basis for trained immunity. Science 345, 1250684 (2014).

35. Saeed, S. et al. Epigenetic programming of monocyte-to-macrophage differentiation and trained innate immunity. Science 345, 1251086 (2014).

36. Shon, W.J, Lee, Y.K., Shin, J.H., Choi, E.Y. \& Shin, D.M. Severity of DSSinduced colitis is reduced in Ido1-deficient mice with down-regulation of TLR-MyD88-NF-kB transcriptional networks. Sci. Rep. 5, 17305 (2015).

37. Takahashi, K., Hayashi, N., Shimokawa, T., Umehara, N., Kaminogawa, S. \& $\mathrm{Ra}, \mathrm{C}$. Cooperative regulation of $\mathrm{FC}_{\mathrm{C}}$ receptor gamma-chain gene expression by multiple transcription factors, including Sp1, GABP, and Elf-1. J. Biol. Chem. 283, 15134-15141 (2008). 